\title{
Characteristics of colliding density currents: a numerical and theoretical study
}

Article

Accepted Version

Cafaro, C. and Rooney, G. G. (2018) Characteristics of colliding density currents: a numerical and theoretical study. Quarterly Journal of the Royal Meteorological Society, 144 (715). pp. 1761-1771. ISSN 1477-870X doi: https://doi.org/10.1002/qj.3337 Available at https://centaur.reading.ac.uk/77904/

It is advisable to refer to the publisher's version if you intend to cite from the work. See Guidance on citing.

To link to this article DOI: http://dx.doi.org/10.1002/qj.3337

Publisher: Royal Meteorological Society

All outputs in CentAUR are protected by Intellectual Property Rights law, including copyright law. Copyright and IPR is retained by the creators or other copyright holders. Terms and conditions for use of this material are defined in the End User Agreement.

\section{www.reading.ac.uk/centaur}

\section{CentAUR}

Central Archive at the University of Reading 
Reading's research outputs online 


\title{
Characteristics of colliding density currents: a numerical and
}

\section{theoretical study}

\author{
Carlo Cafaro1 $^{\mathrm{a}}$, Gabriel G. Rooney ${ }^{\mathrm{b}}$ \\ ${ }^{a}$ Department of Mathematics and Statistics, University of Reading, RG6 6AX, United Kingdom \\ ${ }^{\mathrm{b}}$ Met Office, FitzRoy Road, Exeter, EX1 3BP, United Kingdom
}

${ }^{*}$ Correspondence to: Department of Mathematics and Statistics, University of Reading, Whiteknights, PO Box 220, Berkshire, RG6 6AX, UK. Email: carlo.cafaro@pgr.reading.ac.uk

Funding information

EPSRC, Grant/Award Number:EP/L016613/1.

This paper presents a new set of numerical simulations of two colliding density currents in a idealized framework, integrating the Boussinesq vorticity equation in a rectangular bounded domain. These simulations are used to examine the dynamical features of the collision, in the light of recent laboratory experiments. The collision dynamics present various interesting features. Here we have focused on the interface slope at the front of the two unequal density currents and on the maximum height reached by the fluid after the collision. For the secondary triggering of atmospheric convection by colliding cold pools from previous convective events, these may affect the positioning and the momentum of the collision uplift, respectively.

The interface slope has been shown to be dependent on the currents' buoyancy ratio (i.e. the ratio between the density differences of the two fluids with the ambient fluid), whereas the maximum height has no strong dependence, for a given initial current depth. A theoretical model, based on an analogy with a vortex pair, has been proposed for the interface-slope dependence, taking as input the buoyancy ratio or the propagating speeds. This model agrees reasonably well with the observed numerical values.

Key Words: density currents; collision; vorticity model; parametrization ; fluid dynamics.

This article has been accepted for publication and undergone full peer review but has not been through the copyediting, typesetting, pagination and proofreading process, which may lead to differences between this version and the Version of Record. Please cite this article as doi: $10.1002 /$ qj.3337 


\section{Introduction}

Density currents are primarily horizontal flows generated by a horizontal density gradient. Since they are of practical application to several atmospheric and industrial problems there has been extensive experimental, observational, theoretical and numerical research on density currents, following the early works of von Kármán (1940) and Benjamin (1968). Much of this has been summarised and reviewed by Simpson (1997) and Linden (2012). Thunderstorm outflows and sea breezes have been shown to resemble density currents, by laboratory experiments (Simpson 1969), field observations (Mueller and Carbone 1987) and numerical models (Droegemeier and Wilhelmson 1985).

Many theoretical models, based on energy conservation theory, have considered only irrotational flows. However it is well-known that horizontal density gradients lead to baroclinic vorticity generation (Simpson and Linden 1989), and density currents may also be modelled using a vorticity formulation (e.g. Kay |1992, Liu et al. 2003). Rotunno et al. (1988) used the vorticity formulation to model the phenomenon of cold pools in shear flows. Xu and Moncrieff (1994) also recognized the "importance of vorticity dynamics for the local flow in the vicinity of a density current". Furthermore Nasr-Azadani and Meiburg (2016) have developed a vorticity-based model of quasi-steady and supercritical $(\mathrm{Fr}>1)$ gravity currents.

The interaction between density currents has also received some interest because, for instance, in the atmosphere the collision between mesoscale fronts can be an important trigger for atmospheric convection (Findlater 1964). Intrieri et al. (1990) used lidar field observations to examine aspects of three different colliding thunderstorm outflows. These revealed large vortex rolls and smaller instabilities in the leading edge of the outflows. In addition, in all cases the colder, more dense outflow passed under the warmer outflow, making the warmer air mass the primary source of the updraft. All these collisions initiated new convection. The collision

of sea breezes has been observed by Lapworth (2005) among others, and convective initiation due to colliding land breezes has been modelled by Wapler and Lane (2012).

More recently, van der Wiel et al. (2017) have performed laboratory experiments on colliding density currents with different densities and depths. These parameters have an important influence on the characteristics of the collision (see also Shin et al.|2004; Warren|2014). As discussed by van der Wiel et al. (2017), even the simple case of (quasi) two-dimensional collision in a uniform environment has difficult to parametrise with an analytical model. Hence, we concentrate on this basic case here. In the lower atmosphere, wind Shear (Xu|1992, Parker 1996, Liu and Moncrieff|1996;: Xue et al.|1997, Lee and Wilhelmson|1997, Bryan and Rotunno 2014), ambient stratification (Liu and Moncrieff 2000, Seigel and van den Heever 2012) and three-dimensional front structure (Droegemeier and Wilhelmson 1985, Orf et al. 1996, Vermeire et al.2011) may all modify the cold-pool collision process and effects. These additional processes may begin to be included once the basic collision dynamics are described.

We show herein that the dynamics of two colliding density currents can be reproduced with an idealized two-dimensional, vorticitybased model. In $\S 2$ we replicate numerically similar situations to those of the laboratory experiments of van der Wiel et al. (2017). In particular we focus on the pre-collision and collision stages. In $\S 3$ we discuss some theoretical approaches to predict and explain the shape and orientation of the frontal interface, and compare the predictions with the numerical simulations.

\section{Numerical simulations}

The laboratory experiments have been reproduced numerically using the numerical software package Hydra (http:// www-vortex.mcs.st-and.ac.uk/software.htm] Hydra solves the Boussinesq-Euler equations for the flow of an incompressible fluid in two dimensions, vertical and horizontal. Buoyancy (also known as reduced gravity) is approximately conserved following the flow, up to the action of numerical diffusion. The distribution of buoyancy governs the creation of baroclinic vorticity, and 
the vorticity redistributes the buoyancy. Numerical solution is by the combined Lagrangian advection method, (CLAM, see Dritschel and Fontane 2010, for more details), which preserves the small-scale flow structures at high accuracy. This allows the problem of density-current collision to be recreated in an idealized framework, with minimal diffusion and no possibility of along-front variation. Hence, the basic processes may be isolated and quantified.

The dynamics produce entrainment and mixing as would be expected, so that fluid elements of different densities can mix quite thoroughly. There is no parametrised diffusion to homogenize the densities at the grid scale following this mixing, although numerical diffusion will act in this manner to some extent. Despite these simplifications, over the relatively short timescales of these simulations,

the resultant dynamics are expected to still represent the significant features of the flow, and the numerical results closely resemble the flows in the laboratory experiments of van der Wiel et al. (2017), as shown in the following and in the animations presented as supplementary material. Results from the numerical model have also been compared previously with experiments on billows in stratified flow by Carr et al. (2017).

\subsection{Model description and initial configuration}

2

Hydra solves the following dimensionless equation set on a two-dimensional (vertical and horizontal) rectangular domain, where overbars denote dimensionless variables,

$$
\begin{aligned}
\frac{D \bar{\eta}}{D \tau} & =\frac{\partial \bar{b}}{\partial \bar{x}} \\
\frac{D \bar{b}}{D \tau} & =0 \\
\bar{\nabla} \cdot \overline{\mathbf{u}} & =0,
\end{aligned}
$$

Where $\mathbf{u}=(u, 0, w)$ is the velocity field in two dimensions. $\eta$ is the vorticity component normal to the plane of the flow, all other components being zero. The dimensionless time, horizontal coordinate and vertical coordinate are denoted $\tau, \bar{x}$ and $\bar{z}$ respectively. Their relationships to the equivalent dimensional variables $t, x$ and $z$, and the non-dimensionalization of the flow variables, will be set below. There is a free-slip boundary condition at all the boundaries of the rectangular domain. The domain is of height $H$ and length $L$. Initially, the two patches of fluid denser than the background occupy regions in the lower corners at either side of the domain, length $l$ and depth $D$ (i.e. the same for each current), see for example figure 1

The current on the 1.h.s. is denoted current 1 and the other is denoted current 2 . The buoyancy is given by $b=g \Delta \rho / \rho_{0}$, where $\Delta \rho=\rho_{0}-\rho, \rho$ is the density and $\rho_{0}$ is the uniform ambient fluid density away from the denser patches. The initial density scales are in proportion $\rho_{0} \leq \rho_{1} \leq \rho_{2}$. That is, current 2 is the more dense.

A uniform ambient density reduces the complexity of the system, since there is no influence of ambient stratification to consider. Its counterpart in the real atmosphere is the deep, well-mixed atmospheric boundary layer, such as occurs in the afternoon on days prone to convective activity. It is therefore also a suitable background state for idealisations of convective cold-pool collisions.

The precise initial distribution of the buoyancy (see figure 1) is specified as follows:

$$
\bar{b}(\bar{x}, \bar{z})=\left[-\frac{1}{2} b_{1} \cdot \operatorname{erfc}\{(\bar{x}-l) / \varepsilon\}-\frac{1}{2} \cdot b_{2} \operatorname{erf}\{(\bar{x}-L+l) / \varepsilon\}-\frac{1}{2} b_{2}\right] \cdot \phi(\bar{z})
$$

where

This article is protected by copyright. All rights reserved $(\bar{z})=\frac{1}{2} \operatorname{erfc}\{(\bar{z}-D) / \varepsilon\}$, 
iN 8

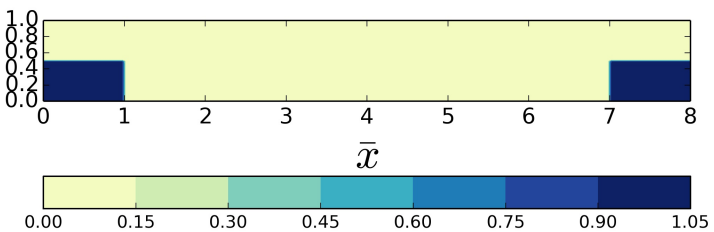

Figure 1. Initial $(\tau=0)$ distribution of the absolute value of the buoyancy, $|\bar{b}(\bar{x}, \bar{z})|$, for simulation 6 as in table $\mathrm{I}$

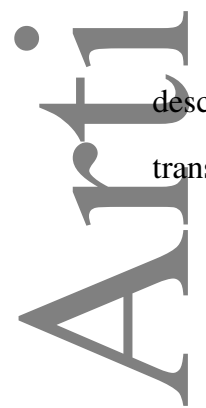

are the error function and its complementary, respectively.

$$
\operatorname{erf}(x)=\frac{1}{\sqrt{\pi}} \int_{-x}^{x} e^{-s^{2}} d s, \quad \operatorname{erfc}(x)=1-\operatorname{erf}(x)
$$

The variables are non-dimensionalized relative to the denser current,

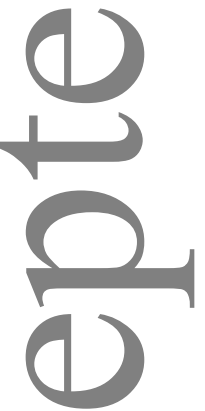

$$
\begin{aligned}
& \bar{x}=x / l, \quad \bar{z}=z / l \\
& \tau=t / \sqrt{l /\left|b_{2}\right|} \\
& \bar{\eta}=\eta / \sqrt{\left|b_{2}\right| / l} \\
& \bar{b}=b /\left|b_{2}\right| \\
& \overline{\mathbf{u}}=\mathbf{u} / \sqrt{\left|b_{2}\right| l}
\end{aligned}
$$

The numerical solution will depend on the dimensionless parameters $D / H, l / L, H / L$ and $r_{g}=b_{1} / b_{2}$. Here, simulations for different

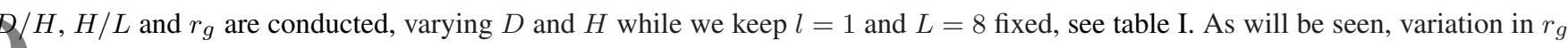
as achieved by keeping $b_{2}$ fixed and varying $b_{1}$.

Simulations $25-27$ on the square domain had a horizontal resolution of 256 points, and all others had a horizontal resolution of 512 points. The vertical resolution was in proportion, according to the domain aspect ratio $H / L$, so as to obtain square grid cells; thus 256 points for simulations $25-27,64$ points for simulations $1-5$, etc. The numerical Reynolds number may be estimated from the grid resolution to be $R e_{\text {num }} \gtrsim 10^{6}$ (Carr et al. 2017, $\S 4.2 .2$ ), bearing in mind that these simulations are two-dimensional.

\subsection{Results}

In this section we present the numerical simulation results for the pre-collision (see figure 2) and collision phases. Animations of some of the simulations are available as supplementary material. For quantitative analysis, we concentrate on the partial-depth simulations, since these whifle is motected by conyright. Adl rights reserved. flows in the lower atmospheric boundary layer. 


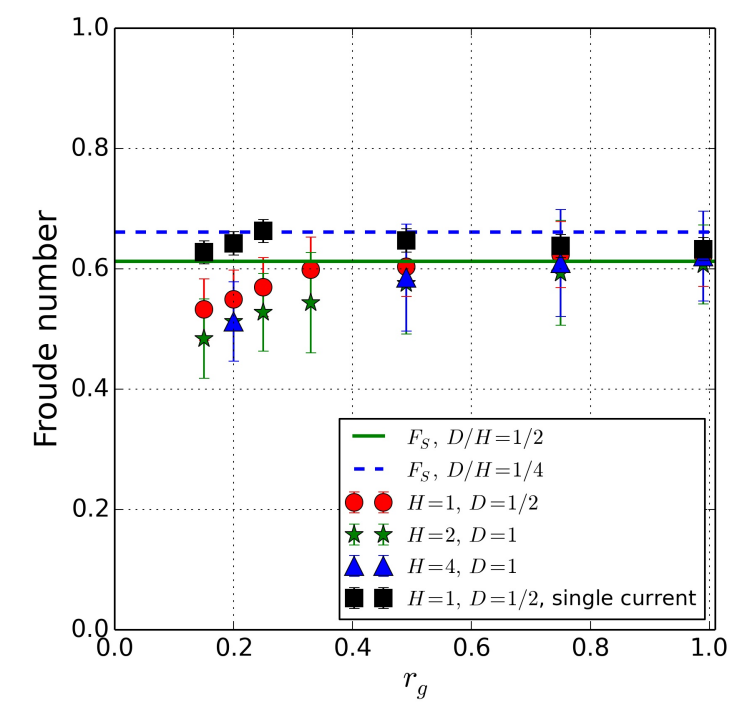

Figure 3. Froude numbers versus $r_{g}$. Error bars show one standard deviation with respect to the mean of density current speeds for different time steps. Continuous lines are theoretical values based on 13 . The simulations are a subset of those listed in table $\mathrm{I}$

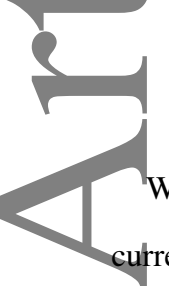

We have calculated (12) also for the single density current case, for $H=1$ and $D=1 / 2$, as also shown in figure 3 The singleurrent simulations are initialised as the two-current simulations but omitting the dense fluid on the r.h.s. In the single-current case the current propagates faster than if there is a current on the other side, as is shown by the Froude number being greater by up to approx. $16 \%$ in these experiments. This means that in the two-current case, the presence of the second current exerts a small influence on the propagation of the first current, even before collision. It is expected that this effect is greater here, in a purely two-dimensional domain 4 of limited extent, than it would be in the atmospheric boundary layer.

Given that the single-current speeds appear to be more uniform with $r_{g}$, the slight fall-off of the speed of current 1 at lower $r_{g}$ values seems to be an effect of having an oncoming density current of significantly greater momentum, therefore presumably driving a relatively stronger opposing flow in the ambient.

\subsubsection{Collision characteristics}

In this section we present the numerical results for the collision of two density currents. The numerical simulations presented here are able to reproduce the main features of the collisions observed in the laboratory experiments of van der Wiel et al. (2017). For direct comparison between the numerical simulations and laboratory experiments, see the supplementary videos S1 and S3, to be compared with with videos S1 and S2 of van der Wiel et al. (2017).

Figure 4 shows Hovmöller diagrams based on the lowest level of the gridded model output for simulations 13 and 16. Before the collision the two currents propagate at certain speed (which we have found different from the single current case, as shown in the previous section). After the collision the denser current propagates at slightly slower rate than before the collision, for the non symmetric case $\left(r_{g}=0.33\right)$. Also, there is mixing occurring in the collision zone. This is in agreement with van der Wiel et al. (2017), fig. 8 .

For comparison with the Hovmöller plots, and with the experiments of van der Wiel et al. (2017), examples of the buoyancy and vorticity fields during symmetric and asymmetric collisions are shown in figure 5 The vorticity distributions in these cases show a significant difference at the interface. Namely, in the symmetric case (figure 5b the frontal vortex sheets combine into a vortex pair that propagates vertically upward, whereas in the asymmetric case (figure 5d) the stronger vortex sheet at the front of the more dense (r.h.s.) Chis article is protected by coptright Adll rights reserved. 

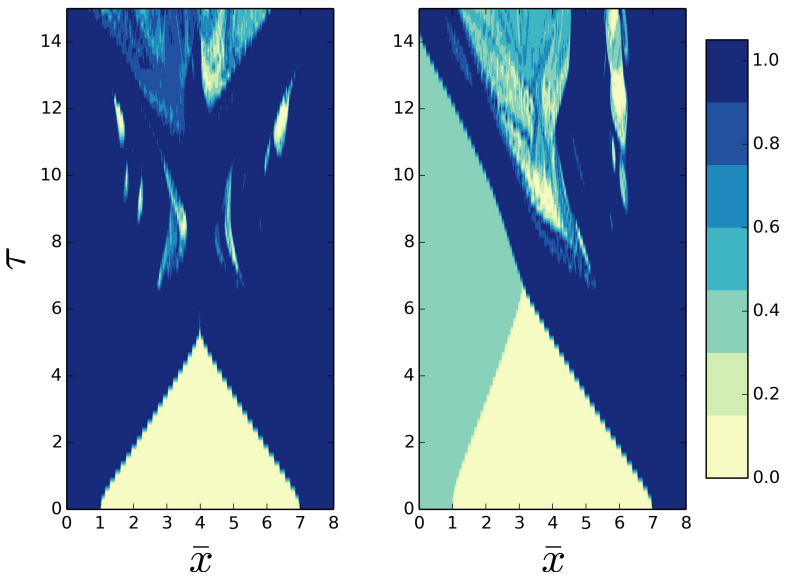

Figure 4. Hovmöller diagrams for simulation 13 (left) and simulation 16 (right) at height $\bar{z}=0$.

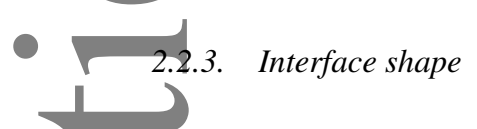

The interface shape may be characterized to some extent by its angle $\gamma$ to the horizontal. Figure 5 indicates that the angle is not constant with respect to time, and figure 6 shows that the interface shape becomes more complex at later times. Nonetheless, the measured angle at a particular dimensionless time post collision indicates how the interface varies from one simulation to another.

The angle between the two fluids, with respect to the horizontal line is given by:

$$
\gamma=\arctan \left(\frac{\Delta z}{\Delta x}\right)
$$

The algorithm to calculate the angle is based on finding the coordinates of discontinuities at two different vertical levels in the buoyancy profiles (i.e. where the two fluids meet). The vertical levels were taken from the interface, identified by the buoyancy discontinuity, in height range between $0<\bar{z}<D / 2$. Three points in the upper part of this range were considered along with two points in the lower part, to generate six values of the interface angle. From these, a mean and standard deviation were calculated. We calculated the angle at time $\tau=0.5$ after the collision. The results are shown in figure 7 , for different $r_{g}$ and for different $H$ and $D$. It can be seen that the

angle does not have a clear dependence on $D / H$, although generally increases with increasing $r_{g}$.

\subsubsection{Collision maximum height}

Another important feature of the collision is the maximum height reached by the two fluids. In an atmospheric context, this may be a significant factor in whether colliding cold-pool fronts are able to initiate new convective cells.

In the symmetric case $r_{g}=0.99$, the front is almost vertical (figures 5c, 7p), while in the asymmetric cases $\left(r_{g}<0.99\right)$ the front is tilted. For later time steps in the non-symmetric case the denser fluid propagates underneath the less dense fluid, compare figures $5 \mathrm{a}$ and 5c Recall that this type overlapping was also observed by Intrieri et al. (1990), and while it means that the perturbation height may be insensitive to $r_{g}$, it has a possible bearing on the properties at the source of the updraft. To illustrate this interaction, figure 6 shows the simulation evolution after the collision. It can be seen that the maximum height reached by the fluid in the symmetric case (figure 6a is comparable to the maximum height in the asymmetric case (figure 6b.

This figure also shows the spatio-temporal domain over which the maximum height has been calculated. This is done by counting the number of gridboxes $G$ at each level where the the absolute value of the buoyancy is above a threshold of $0.8 r_{g}$, which takes into account that different buoyancies may be present. In general $G$ decreases with height. Then the maximum height is defined as the height at this artichle is protected by copvright Adl rights reserved. 


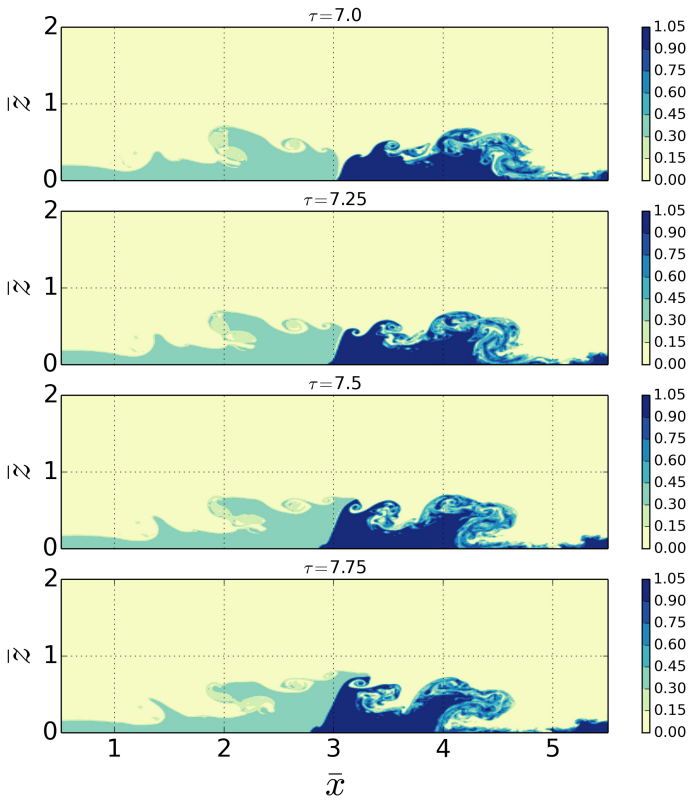

(a) Buoyancy $r_{g}=0.33$

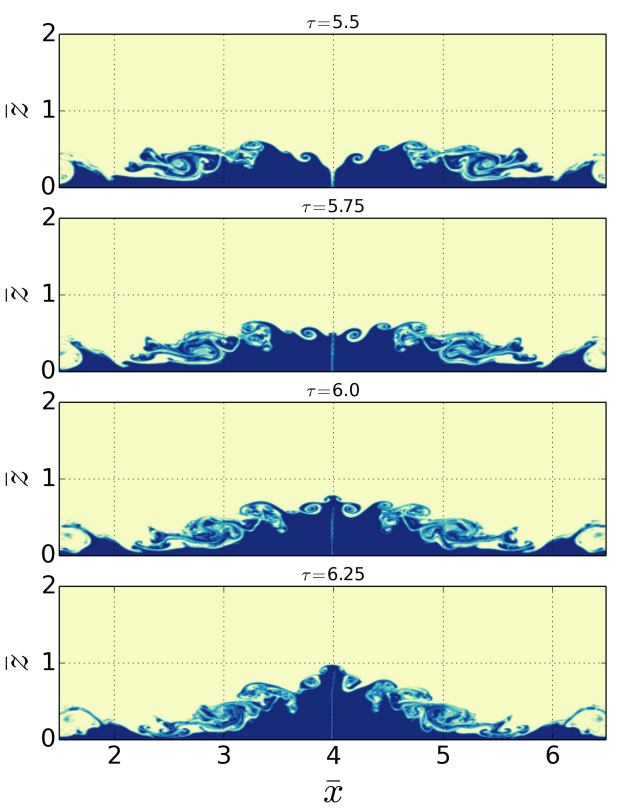

(c) Buoyancy $r_{g}=0.99$

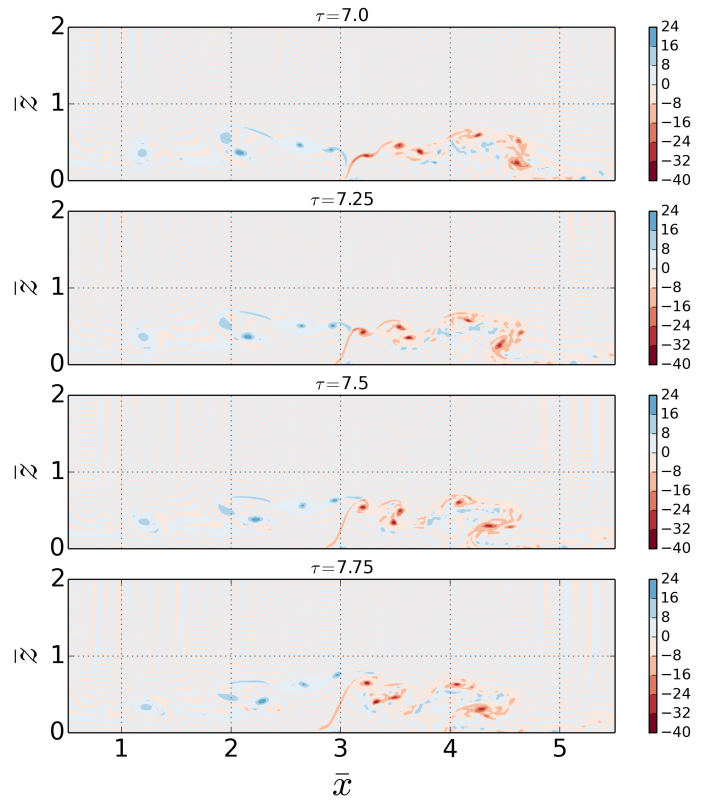

(b) Vorticity $r_{g}=0.33$
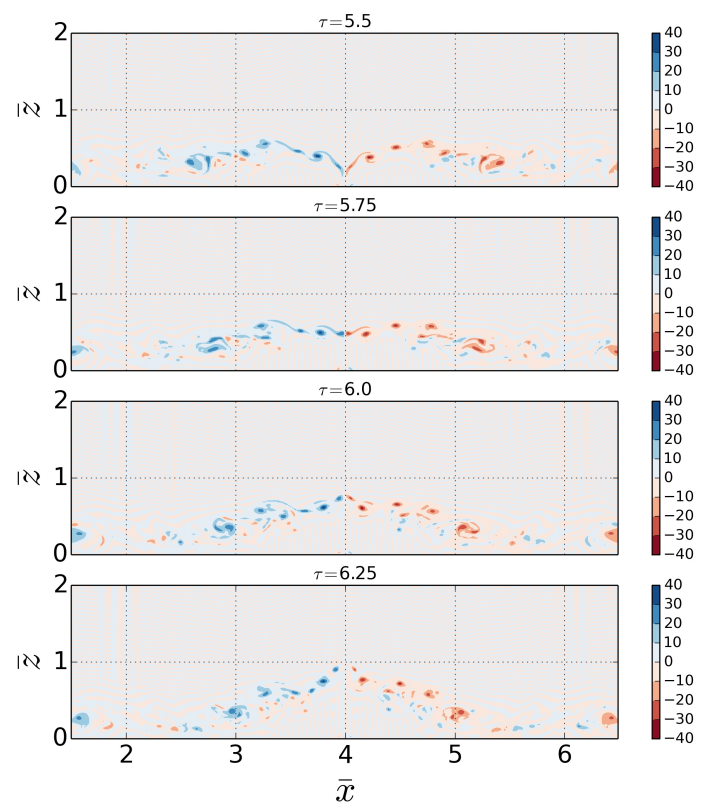

(d) Vorticity $r_{g}=0.99$.

Figure 5. Time sequence of buoyancy and vorticity profiles for two different $r_{g}$ from the moment of collision. The simulations shown are the same of figure 4

The results of this calculation are shown in figure 8 Since we vary both $D / H$ and $H / L$ in these simulations, the results are plotted in two ways. Figure $8 \mathrm{a}$ shows the height normalized by the domain height $H$ when $D / H<1$ is fixed but $H / L$ varies. Figure $8 \mathrm{~b}$ shows the height normalized by the initial current height $D$ when $D / H<1$ varies. Figure $8 \mathrm{c}$ shows the height normalized by the domain height when $D / H=1$. This last case represents the only direct correspondence in parameter space between the numerical simulations and the laboratory experiments of van der Wiel et al. (2017), whose results are also plotted for comparison.

As previously found by van der Wiel et al. (2017), it is not possible to infer any strong relationship between the maximum collision height and $r_{g}$. Figures $8 \mathrm{a}$ and $8 \mathrm{~b}$ show that, for these data, the collision height seems to scale primarily with $D$. Figure $8 \mathrm{~b}$ shows that, for $D / H \leq 1 / 2$, increasing the relative height of the domain does not greatly affect the collision height, relative to the initial current This artigle is effected by gopvright All rights reserved. 


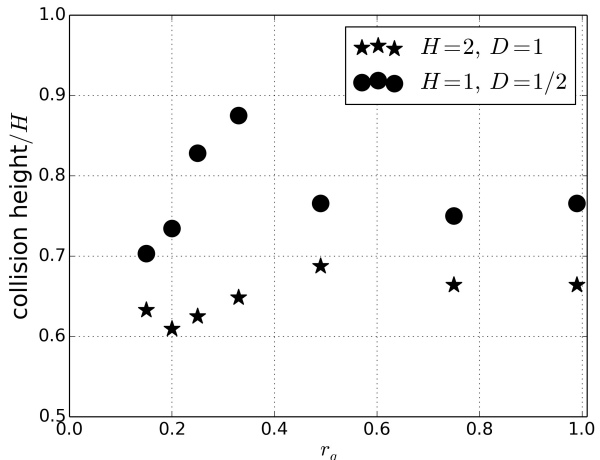

(a) Numerical simulations $D / H=1 / 2$.

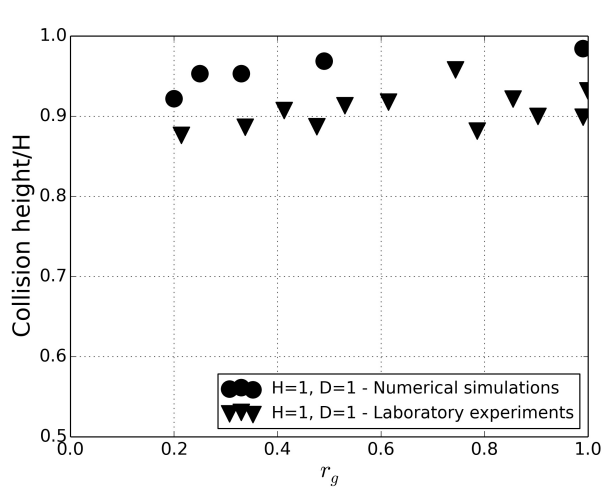

(c) Full-depth numerical simulations compared with the full-depth laboratory experiments of van der Wiel et al. (2017).

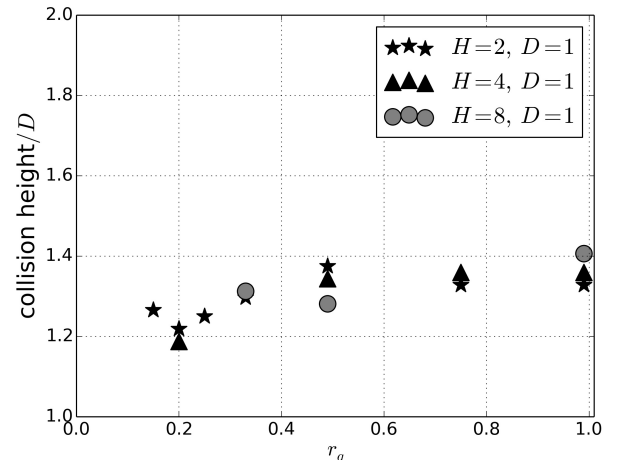

(b) Numerical simulations $D=1, H=\{2,4,8\}$.
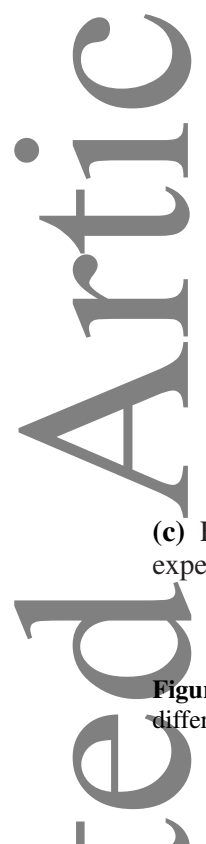

Figure 8. Comparison of maximum collision heights: (a) numerical simulations with $D / H=1 / 2$ for two values of $H$, (b) numerical simulations with the same $D$ but different $H$, and (c) comparison of full-depth $(H=D=1)$ maximum collision heights between numerical simulations and laboratory experiments.

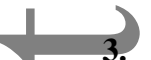

Theoretical considerations

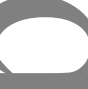

In the previous sections we have looked at the output of the numerical model solving (13), in particular in figure (7) the variation of collision angle with the density ratio $r_{g}$ of the two density currents. In this section we present a model of the collision angle $\dagger^{\dagger}$ based On the properties of the two incoming density currents. This will aid the future parametrization of these processes in large-scale models which are unable to resolve the details of such flows.

The similarity between the numerical results presented here and the experiments of van der Wiel et al. (2017) indicates that the idealized equations capture the essential features of the flow. Since the numerical model has buoyancy and vorticity as its dynamic prognostic variables, this indicates that the baroclinic vorticity plays a crucial role in the front and collision dynamics. Thus the flow been modelled in terms of the vortex dynamics.

For a steadily translating density current, the vorticity generation is balanced to first order by the horizontal current motion. We conjecture that during a density-current collision, this balance is lost, and takes some time to be recovered. This implies that the dynamics during the collision are controlled by the motion of the current-head vortices which are already established.

Note that in the rest of this section we are concerned with the collision and its aftermath, hence time is taken as relative to the moment of collision. That is, $\tau=0$ is re-defined as the collision moment, identified as the first contact of the density currents at the lowest level. 


\subsection{Vortex-pair model}

It is well-known (e.g. Lamb 1945, Article 155) that two isolated line vortices rotate around the centre of their system with angular velocity

$$
\Omega=\frac{\Gamma}{2 \pi \delta^{2}}
$$

where $\Gamma$ is the sum of the vortex circulations and $\delta$ is the distance between them. For vortices with different-signed circulations, the density currents are likened to regions of two-dimensional vorticity, considering the shear across the current indicates that the magnitude of the circulation is proportional to the product of the current speed and head height, $\Gamma_{i} \propto U_{i} h$. When equal-height currents interact in a collision, therefore, the net angular velocity of fluid in the collision zone may be approximated by

$$
\Omega \propto \frac{U_{2}-U_{1}}{h}
$$

\section{1}

assuming that the separation of the centres of circulation is also proportional to the head height, i.e. $\delta \sim h$. For two vortices, the net circulation sets the direction of rotation of the pair. Likewise, the direction of the circulation here will be in the same sense as the circulation in the faster i.e. denser current. This implies that the relatively less-dense current will move over the top of the denser current, as is observed.

The currents have very similar front shapes and heights prior to collision so we assume that the collision interface starts from the vertical, and that the angle will evolve simply as the product of $\Omega$ and time. This gives the front angle $\beta$ to the vertical at time $t$ after collision as

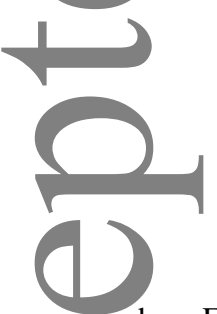

$$
\begin{aligned}
\beta & =k_{0} \frac{U_{2}-U_{1}}{h} t \\
& =k_{0} \operatorname{Fr} \sqrt{b_{2}}\left(1-\sqrt{b_{1} / b_{2}}\right) \frac{t}{\sqrt{h}} \\
& =k_{0} \operatorname{Fr}\left(1-r_{g}^{1 / 2}\right) \tau\left(\frac{l}{h}\right)^{1 / 2}
\end{aligned}
$$

where Fr is the Froude number defined in 12 and $k_{0}$ is an unknown proportionality constant which absorbs the ratio $h / D \approx 0.5$ in the Fr definition. We have assumed that, with the Boussinesq approximation, the Froude numbers of the two currents are approximately equal, which is a common assumption, see for example (13). (Here they have small differences in the range 0-0.1.)

This model is approximate, and is only expected to apply for a short interval during the collision. Nonetheless, it has two implications. The first, from (15), is that, at a given dimensionless time after collision, the angle is proportional to the net circulation in the collision zone. This follows fairly immediately from the application of this type of model. The second, from (17), is a parabolic dependence of the measured angle on the density ratio, with a vertical interface in the case $r_{g}=1$.

The first of these relationships may be tested by considering the total, or net, vorticity in a region of the simulation domain (always of the same area) at a particular time after the collision, and covering the point of collision. An example of the vorticity evolution in such a region is shown in figure 9

Figure 10 shows how this net vorticity, taken to be equivalent to the circulation per unit (constant) area of the box over which the vorticity is summed, varies with the mean interface angle measured at the same time. In this plot the net vorticity is multiplied by the time interval since the collision to correspond more closely to 17 . It can be seen that the data appear to follow a general linear trend with increas article is protected by gopyright All rights reserved the model prediction. 
For the second, the angle to the vertical at any particular time $\tau$ is given by (17). Taking $\tau=0.5$ the angle to the horizontal is then

$$
\gamma=\frac{\pi}{2}-\beta=\left(\frac{\pi}{2}-k\right)+k r_{g}^{1 / 2}
$$

The coefficient

$$
k=\frac{k_{0}}{2} \operatorname{Fr}\left(\frac{l}{h}\right)^{1 / 2}
$$

(c)

may be determined from the numerical results at $\tau=0.5$.

Figure 7 shows the comparison of the theory with the numerical values. The relatively small range of $3 / 2 \leq k \leq 5 / 2$ covers most of he spread of angles calculated numerically, hence a value of $k=2$ may be taken as representative. Hence

$$
k_{0} \approx 4 \mathrm{Fr}^{-1}\left(\frac{l}{h}\right)^{-1 / 2}
$$
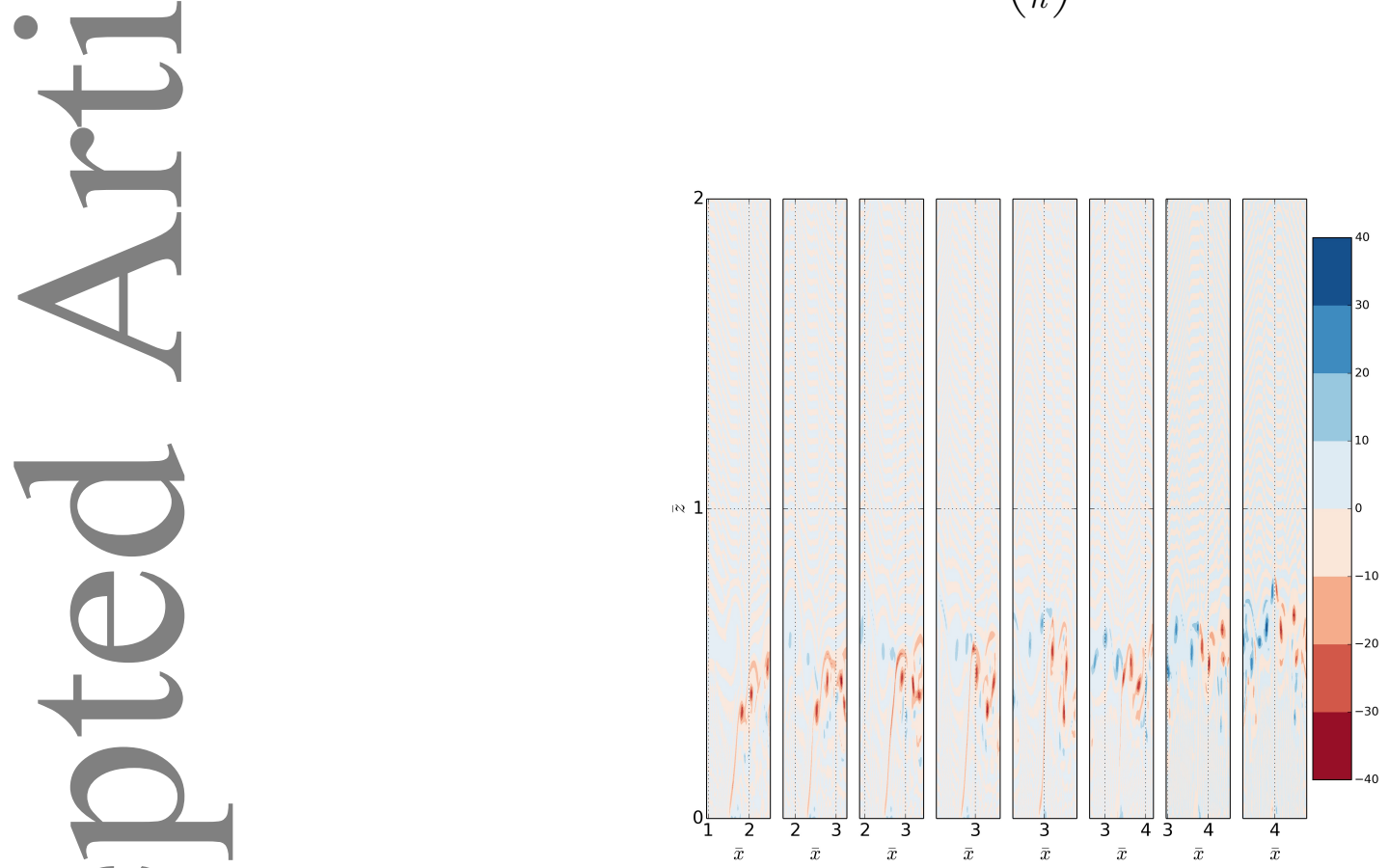

Figure 9. Snapshot of the vorticity field 2 output time steps after the collision for different $r_{g}$ (simulations $13-20$ in order from right to left), in the two dimensional domain where we have integrated $\eta$ as in figure 10 The width of the domain is the same for all $r_{g}$, and equal to $0.2 L$.
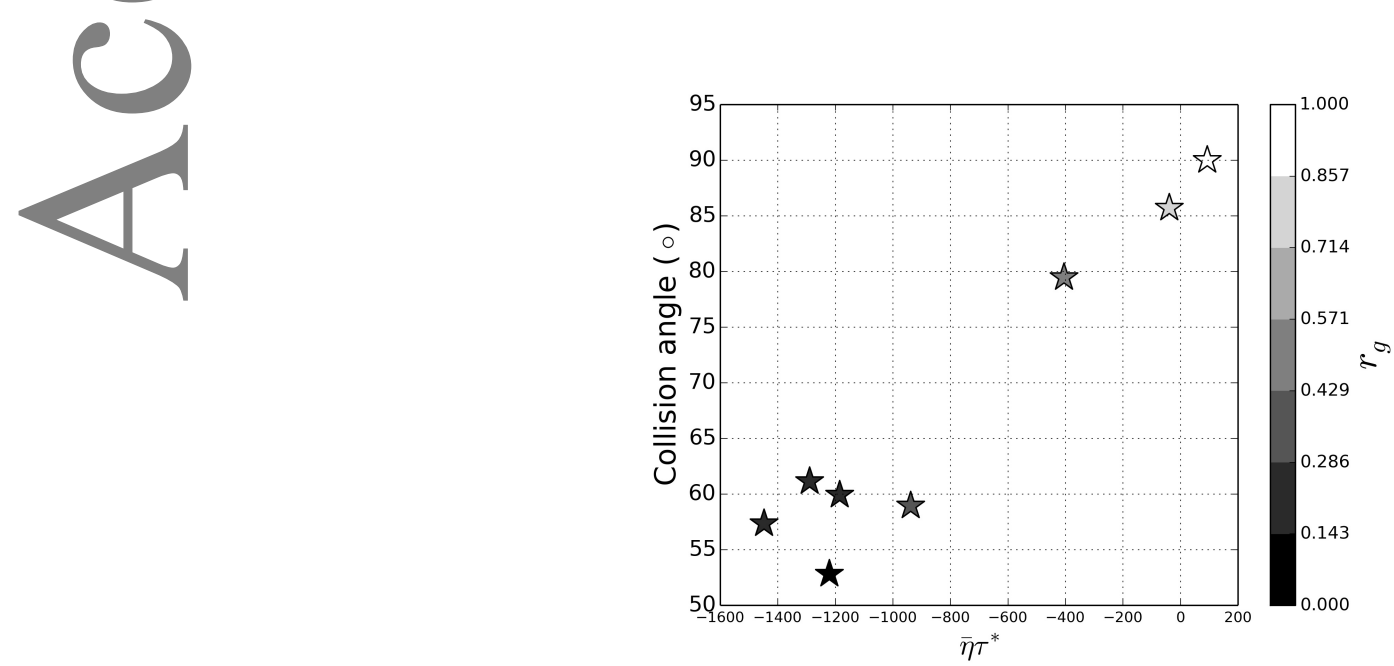

Figure 10. Relationship between the integral of the vorticity in the $2 \mathrm{D}$ box as described in figure 9 at $\tau^{*}=0.5$ post-collision and the collision angle measured for simulations $13-20$ as in table I with a colorbar representing $r_{g}$.

This article is protected by copyright. All rights reserved. 
This simple model of the angle dependence obtained from approximating the vorticity dynamics would not seem to be derivable from considerations of conservation of energy or momentum alone, but despite its simplicity and approximate nature it fits the data reasonably well. Also, as has been shown, the present results from a numerical model in which the main variables are vorticity and buoyancy reproduce many of the features of the experiments of van der Wiel et al. (2017) (see also the supplementary flow animations). This demonstrates that the consideration of vorticity is crucial in fully understanding the dynamics of colliding density currents.

\section{Conclusions and future work}

have performed numerical simulations of two colliding density currents in a limited rectangular domain for different buoyancy ratios $r_{g}$. These have been based around the recent laboratory experiments performed by van der Wiel et al. (2017). Beside the buoyancy ratios, we have also varied the height of the domain and the relative current depth, to investigate the effect of the rigid top lid and so to make the simulation closer to a realistic atmospheric situation. We have considered only currents of equal initial depth, and of one initial length relative to the domain length, to keep the parameter space to manageable proportions, and to simplify the modelling requirement. Investigating the other parameters could be the subject of future work.

We have looked at the slope of the collision front that forms since the denser current deflects the other fluid upward, and also at the maximum height reached by the fluid raised after the collision. While we have found a large variation in the initial angle of collision with $r_{g}$, this is not the case for the maximum collision height. This is consistent with an interpretation of the front collision being governed by the interaction of the vortex sheets at the current heads, which leads to unequal currents tending to circulate around one another rather than to "splash" upward. We remark that this has possible relevance in determining which current interacts with the source of any resulting convective updraft.

As noted in the introduction however, while the collision height is insensitive to $r_{g}$ in the basic case, other factors may produce

Secondary effects. For instance, the position or maximum of collision height may vary if the fronts have appreciable curvature (Droegemeier and Wilhelmson 1985), and the height perturbation may be transmitted vertically and horizontally by gravity waves in a stratified environment (Liu and Moncrieff 2000). Further work in this area could involve the investigation of stratification effects

(a)so possible with Hydra), or wind-shear and front-curvature effects (perhaps with a large-eddy model).

A theoretical model, based on a vortex-pair argument, has been proposed to predict the interface slope at the front, taking as input either the buoyancy ratio or the relative propagation speeds of the currents. This has been shown to agree well with the results of the simulations.

In the wider context of convection parametrization, increasing model resolution (in time and space) produces a requirement for better understanding of the dynamics, rather than merely the thermodynamics, of atmospheric convection. Hence, triggering by convective cold pools has become an area of current interest (e.g. Grandpeix and Lafore 2010, Rio et al. 2013), since it represents one means by which a dynamical "memory" prolongs the convective state. One proposed mechanism for cold pools overcoming convective inhibition is kinematic uplift, either at the front of an advancing cold pool or where cold pools collide. It has been suggested recently (Torri et al. 2015, see also references therein) that this is the most important process for initiating parcel ascent from the surface layer, with thermodynamic processes reinforcing the convective motion at higher levels. The insights into collision dynamics obtained here will be useful in designing the future parametrization of the distribution, in position and strength, of collision-induced convective triggering.

\section{acknowledgements}

CC acknowledges Engineering and Physical Sciences Research Council Mathematics of Planet Earth Centre for Doctoral Training (grant reference EP/L016613/1) and the National Centre for Earth Observation for funding this project.

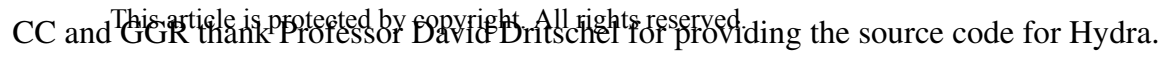


CC has also benefited from fruitful discussions with Dr Michael Whitall about the vorticity model. CC thanks Tom Frame and John Methven for useful comments and suggestion that have improved the manuscript.

Finally the authors acknowledge two anonymous reviewers whose comments led to a very much improved manuscript.

\section{Supporting information}

The following supporting information is available as part of the online article:

Video S1. Movie of the time evolution of buoyancy for simulation 1.

Video S2. Movie of the time evolution of vorticity for simulation 1 .

Video S3. Movie of the time evolution of the buoyancy for simulation 3.

Video S4. Movie of the time evolution of vorticity for simulation 3.

Video S5. Movie of the time evolution of buoyancy for simulation 13.

Video S6. Movie of the time evolution of vorticity for simulation 13.

Video S7. Movie of the time evolution of buoyancy for simulation 16.

Video S8. Movie of the time evolution of vorticity for simulation 16.

Video S9. Movie of the time evolution of buoyancy for simulation 25.

Video S10. Movie of the time evolution of buoyancy for simulation 27.

Figure S1. Schematic diagram representing the collision moment to explain the terms used in the alternative theoretical model.

Figure S2. Figure representing the comparison between the alternative theoretical model and the numerical values of the interface slope as in figure 7

\section{Conflict of interest}

None.

\section{References}

Benjamin, T. B. (1968) Gravity currents and related phenomena. Journal of Fluid Mechanics, 31, 209-248.

Bryan, G. H. and Rotunno, R. (2014) The optimal state for gravity currents in shear. Journal of the Atmospheric Sciences, 71, 448-468.

Carr, M., Franklin, J., King, S. E., Davies, P. A., Grue, J. and Dritschel, D. G. (2017) The characteristics of billows generated by internal solitary waves. Journal of Fluid Mechanics, 812, 541-577.

Dritschel, D. G. and Fontane, J. (2010) The combined Lagrangian advection method. J. Comput. Physics, $229,5408-5417$.

Droegemeier, K. K. and Wilhelmson, R. B. (1985) Three-dimensional numerical modeling of convection produced by interacting thunderstorm outflows. part i: Control simulation and low-level moisture variations. Journal of the Atmospheric Sciences, 42, 2381-2403.

Findlater, J. (1964) The sea-breeze and inland convection: an example of their interrelation. Meteorological Magazine, 93, 82 - 9.

Grandpeix, J.-Y. and Lafore, J.-P. (2010) A density current parameterization coupled with emanuel's convection scheme. part i: The models. Journal of the Atmospheric Sciences, 67, 881-897.

Intrieri, J. M., Jr., A. J. B. and Hardesty, R. M. (1990) Details of colliding thunderstorm outflows as observed by doppler lidar. Journal of the Atmospheric Sciences, 47, 1081-1099.

von Kármán, T. (1940) The engineer grapples with nonlinear problems. Bull. Amer. Math. Soc., 46, 615-683.

Kay, A. (1992) Frontogenesis in gravity-driven flows with non-uniform density gradients. Journal of Fluid Mechanics, 235, 529-556.

Lamb, H. (1945) Hydrodynamics. Dover Publications.

Lapworth, A. (2005) Collision of two sea-breeze fronts observed in wales. Weather, 60, 316-318.

Lee, B. D. and Wilhelmson, R. B. (1997) The numerical simulation of non-supercell tornadogenesis. Part i: Initiation and evolution of pretornadic misocyclone circulations along a protected by copyright. All rights reseryed. 
Linden, P. (2012) Gravity currents-theory and laboratory experiments. In Buoyancy-Driven Flows (eds. E. Chassignet, C. Cenedese and J. Verron), 13-51. Cambridge University Press.

Liu, C. and Moncrieff, M. W. (1996) A numerical study of the effects of ambient flow and shear on density currents. Monthly Weather Review, 124, $2282-2303$. — (2000) Simulated density currents in idealized stratified environments. Monthly Weather Review, 128, 1420-1437.

Liu, J.-G., Wang, C. and Johnston, H. (2003) A fourth order scheme for incompressible boussinesq equations. Journal of Scientific Computing, 18, $253-285$.

Mueller, C. K. and Carbone, R. E. (1987) Dynamics of a thunderstorm outflow. Journal of the Atmospheric Sciences, 44, 1879-1898.

Nasr-Azadani, M. M. and Meiburg, E. (2016) Gravity currents propagating into ambients with arbitrary shear and density stratification: vorticity-based modelling. Quarterly Journal of the Royal Meteorological Society, 142, 1359-1370.

Orf, L. G., Anderson, J. R. and Straka, J. M. (1996) A three-dimensional numerical analysis of colliding microburst outflow dynamics. Journal of the Atmospheric Sciences, 53, 2490-2511.

Parker, D. J. (1996) Cold pools in shear. Quarterly Journal of the Royal Meteorological Society, 122, 1655-1674.

Rio, C., Grandpeix, J.-Y., Hourdin, F., Guichard, F., Couvreux, F., Lafore, J.-P., Fridlind, A., Mrowiec, A., Roehrig, R., Rochetin, N., Lefebvre, M.-P. and Idelkadi, A. (2013) Control of deep convection by sub-cloud lifting processes: the alp closure in the lmdz5b general circulation model. Climate Dynamics,

40, 2271-2292.

Rottman, J. W. and Simpson, J. E. (1983) Gravity currents produced by instantaneous releases of a heavy fluid in a rectangular channel. J. Fluid Mech., 135, 95-110.

Rotunno, R., Klemp, J. B. and Weisman, M. L. (1988) A theory for strong, long-lived squall lines. Journal of the Atmospheric Sciences, 45, 463-485.

eigel, R. B. and van den Heever, S. C. (2012) Simulated density currents beneath embedded stratified layers. Journal of the Atmospheric Sciences, 69 , 2192-2200.

Shin, J. O., Dalziel, S. B. and Linden, P. F. (2004) Gravity currents produced by lock exchange. Journal of Fluid Mechanics, 521, 1-34.

Simpson, J. E. (1969) A comparison between laboratory and atmospheric density currents. Q. J. R. Met. Soc., 95, 758 - 765.

(1997) Gravity Currents in the Environment and the Laboratory. Second Edition. Cambridge University Press.

Simpsor pson, J. E. and Linden, P. F. (1989) Frontogenesis in a fluid with horizontal density gradients. Journal of Fluid Mechanics, 202, 1-16.

( Torri,

orri, G., Kuang, Z. and Tian, Y. (2015) Mechanisms for convection triggering by cold pools. Geophysical Research Letters, 42, $1943-1950$.

ermeire, B. C., Orf, L. G. and Savory, E. (2011) A parametric study of downburst line near-surface outflows. Journal of Wind Engineering and Industrial Aerodynamics, 99, 226-238.

Wapler, K. and Lane, T. P. (2012) A case of offshore convective initiation by interacting land breezes near Darwin, Australia. Meteorology and Atmospheric Physics, 115, 123-137.

Warren, R. A. (2014) Quasi-stationary convective systems in the UK. Ph.D. thesis, University of Reading.

der Wiel, K., Gille, S. T., Llewellyn Smith, S. G., Linden, P. F. and Cenedese, C. (2017) Characteristics of colliding sea breeze gravity current fronts: a laboratory study. Quarterly Journal of the Royal Meteorological Society, 143, 1434-1441.

Xu. Q. (1992) Density currents in shear flows-a two-fluid model. Journal of the Atmospheric Sciences, 49, 511-524.

Xu, Q. and Moncrieff, M. W. (1994) Density current circulations in shear flows. Journal of the Atmospheric Sciences, 51, $434-446$.

Xue, M., Xu, Q. and Droegemeier, K. K. (1997) A theoretical and numerical study of density currents in nonconstant shear flows. Journal of the Atmospheric Sciences, 54, 1998-2019. 